\title{
VISUAL TOPOLOGICAL MAP BUILDING IN SELF-SIMILAR ENVIRONMENTS
}

\author{
Toon Goedemé, Tinne Tuytelaars and Luc Van Gool \\ PSI - VISICS \\ Katholieke Universiteit Leuven, Belgium \\ \{tgoedeme,tuytelaa,vangool\}@esat.kuleuven.be
}

\begin{abstract}
Keywords: Mobile robot navigation, Topological map building, Omnidirectional vision, Dempster-Shafer theory.
Abstract: This paper describes a method to automatically build topological maps for robot navigation out of a sequence of visual observations taken from a camera mounted on the robot. This direct non-metrical approach relies completely on the detection of loop closings, i.e. repeated visitations of one particular place. In natural environments, visual loop closing can be very hard, for two reasons. Firstly, the environment at one place can look differently at different time instances due to illumination changes and viewpoint differences. Secondly, there can be different places that look alike, i.e. the environment is self-similar. Here we propose a method that combines state-of-the-art visual comparison techniques and evidence collection based on Dempster-Shafer probability theory to tackle this problem.
\end{abstract}

\section{INTRODUCTION AND RELATED WORK}

In every mobile robot application, the internal representation of the perceived environment is of crucial importance. The environment map is the basis for other tasks, like localisation, path planning, navigation, etc ... The map building field can be divided in two major paradigms: geometrical maps and topological maps, even if hybrid types have been implemented (Tomatis et al., 2002).

In the traditional geometrical paradigm, maps are quantitative representations of the environment wherein locations are given in metrical coordinates. One approach often used is the occupancy map: a grid of evenly spaced cells each containing the information whether the corresponding position in the real world is occupied.

Because the latter is error-prone, time-consuming, and memory-demanding, we chose for the topological paradigm. Here, the environment map is a qualitative graph-structured representation where nodes represent distinct places in the environment, and arcs denote traversable paths between them. This flexible representation is not dependent on metrical localisation such as dead reckoning, is compact, allows highlevel symbolic reasoning and mimics the internal map humans and animals use (Tolman, 1948).
Several approaches for automatic topological map building have been proposed, differing in the method and the sensor(s) used. In our work, we solely use a camera as sensor. We chose for an omnidirectional system with a wide field-of-view.

Other researchers worked mainly with other sensors, such as the popular laser range scanner. A standard approach is the one of (Nagatani et al., 1998), who construct generalised Voronoi diagrams out of laser range data.

Very popular are various probabilistic approaches of the topological map building problem. (Ranganathan et al., 2005) for instance use Bayesian inference to find the topological structure that explains best a set of panoramic observations, while (Shatkay and Kaelbling, 1997) fit hidden Markov models to the data. If the state transition model of this HMM is extended with robot action data, the latter can be modeled using a partially observable Markov decision process or POMDP, as in (Koenig and Simmons, 1996) and (Tapus and Siegwart, 2005). (Zivkovic et al., 2005) solve the map building problem using graph cuts.

In contrast to these global topology fitting approaches, an alternative way is detecting loop closings. During a ride through the environment, sensor data is recorded. Because it is known that the driven path is traversable, an initial topological representa- 
tion is one long edge between start and end node. Now, extra links are created where a certain place is revisited, i.e. an equivalent sensor reading occurs twice in the sequence. This is called a loop closing. A correct topological map results if all loop closing links are added.

In natural environments, loop closing based on mere vision input can be very hard, for two reasons. Firstly, the environment at one place can look different at different time instances due to illumination changes, viewpoint differences and occlusions. A comparison technique that is not robust to these changes will overlook some loop closings. Secondly, there can be different places that look alike, i.e. the environment is self-similar. These would add erroneous loop closings and thus yield an incorrect topological map as well.

In (Wahlgren and Duckett, 2005), the authors detect loops by comparing omnidirectional images with local feature techniques, a robust technique we also adopt. But, there method suffers indeed from selfsimilarities, as we experienced in previous work (Goedemé et al., 2004b).

Also in loop closing, probabilistic methods are introduced to cope with the uncertainty of link hypotheses. (Chen and Wang, 2005), for instance, use Bayesian inference. (Beevers and Huang, 2005) recently introduced Dempster-Shafer probability theory into loop closing, which has the advantage that ignorance can be modeled and no prior knowledge is needed. Their approach is promising, but limited to simple sensors and environments. In this paper, we present a new framework for loop closing using rich visual sensors in natural complex environments, which is also based on Dempster-Shafer mathematics but uses it differently.

We continue the paper with a brief summary of Dempster-Shafer theory in section 2. Then we describe the details of our algorithm in section 3 . Section 4 details our real-world experiments. The paper ends with a conclusion in section 5 .

\section{DEMPSTER-SHAFER}

The proposed visual loop closing algorithm relies on Dempster-Shafer theory (Dempster, 1967; Shafer, 1976) to collect evidence for each loop closing hypothesis. Therefore, a brief overview of the central concepts of Dempster-Shafer theory is presented in this section.

Dempster-Shafer theory offers an alternative to traditional probabilistic theory for the mathematical representation of uncertainty. The significant innovation of this framework is that it makes a distinction between multiple types of uncertainty. Unlike tra- ditional probability theory, Dempster-Shafer defines two types of uncertainty:

- Aleatory Uncertainty - the type of uncertainty which results from the fact that a system can behave in random ways (a.k.a. stochastic or objective uncertainty)

- Epistemic Uncertainty - the type of uncertainty which results from the lack of knowledge about a system (a.k.a. subjective uncertainty or ignorance)

This makes it a powerful technique to combine several sources of evidence to try to prove a certain hypothesis, where each of these sources can have a different amount of knowledge (ignorance) about the hypothesis. That is why Dempster-Shafer is typically used for sensor fusion.

For a certain problem, the set of mutually exclusive possibilities, called the frame of discernment, is denoted by $\Theta$. For instance, for a single hypothesis $H$ about an event this becomes $\Theta=\{H, \neg H\}$. For this set, traditional probability theory will define two probabilities $P(H)$ and $P(\neg H)$, with $P(H)+$ $P(\neg H)=1$. Dempster-Shafer's analogous quantities are called basic probability assignments or masses, which are defined on the power set of $\Theta: 2^{\Theta}=$ $\{A \mid A \subseteq \Theta\}$. The mass $m: 2^{\Theta} \rightarrow[0,1]$ is a function meeting the following conditions:

$$
m(\emptyset)=0 \quad \sum_{A \in 2^{\Theta}} m(A)=1 .
$$

For the example of the single hypothesis $H$, the power set becomes $2^{\Theta}=\{\emptyset,\{H\},\{\neg H\},\{H, \neg H\}\}$. A certain sensor or other information source can assign masses to each of the elements of $2^{\Theta}$. Because some sensors do not have knowledge about the event (e.g. it is out of the sensor's field-of-view), they can assign a certain fraction of their total mass to $m(\{H, \neg H\})$. This mass, called the ignorance, can be interpreted as the probability mass assigned to the outcome ' $H$ OR $\neg H^{\prime}$, i.e. when the sensor does not know about the event, or is - to a certain degree-uncertain about the outcome ${ }^{1}$.

Sets of masses about the same power set, coming from different information sources can be combined together using Dempster's rule of combination:

$$
m_{1} \oplus m_{2}(C)=\frac{\sum_{A \cap B=C} m_{1}(A) m_{2}(B)}{1-\sum_{A \cap B=\emptyset} m_{1}(A) m_{2}(B)}
$$

This combination rule is useful to combine evidence coming from different sources into one set of masses. Because these masses can not be interpreted as classical probabilities, no conclusions about the hypothesis

\footnotetext{
${ }^{1}$ This means also that no prior probability function is needed, no knowledge can be expressed as total ignorance.
} 
can be drawn from them directly. That is why two additional notions are defined, support and plausibility. They are computed as:

$$
\operatorname{Spt}(A)=\sum_{B \subseteq A} m(B) \quad P l s(A)=\sum_{A \cap B \neq \emptyset} m(B)
$$

These values define a confidence interval for the real probability of an outcome: $P(A) \in$ $[\operatorname{Spt}(A), P l s(A)]$. Indeed, due to the vagueness implied in having non-zero ignorance, the exact probability can not be computed. But, decisions can be made based on the lower and upper bounds of this confidence interval.

\section{ALGORITHM}

We apply this mathematical theory on loop closing detection based on omnidirectional image input. Our target application is as follows. A robot is equipped with an omnidirectional camera system and is guided through an environment, e.g. by means of a joystick. While driving around, images are captured at constant time intervals. This yields a sequence of images which the automatic method described in this paper transforms in a topological map. Later, this map can be used for localisation, path planning and navigation, as we described in previous work (Goedemé et al., 2004a) and (Goedemé et al., 2005).

\subsection{Omnidirectional camera system}

The visual sensor we use is a catadioptric system composed by a colour camera and an hyperbolic mirror, as shown in fig. 1. This system is mounted on top of a robot, in our case the electric wheel chair Sharioto. Typical images are shown in fig. 7.

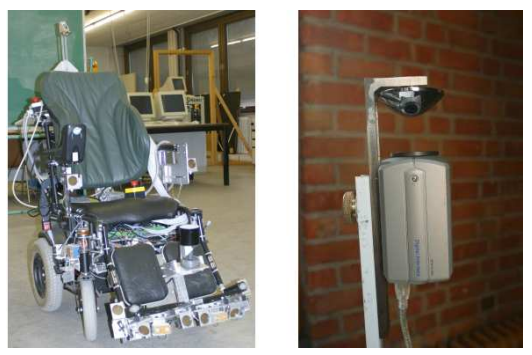

Figure 1: Left: the wheel chair test platform. Right: the omnidirectional camera system.

\subsection{Image comparison}

Because we do not have any other kind of information, the entire topological loop closing is based on images. The target is to find a good way to compare images, such that a second visit to a certain place can be detected as two similar images in the input sequence. As explained before, one of the main challenges is the appearance variation of places. At different time instances (i.e. during different visits), the images acquired at a certain place can vary a lot. This is mostly due to three reasons:

- Illumination differences: The same place is illuminated with a different light source (e.g. somebody switched on a light, the sun emerges from behind the clouds, ...).

- Occlusions: Part of the image can be hidden because of e.g. people passing by.

- Viewpoint differences: It can never be guaranteed that the robot comes back to exactly the same position. Even for small viewpoint changes the image looks different.

We want to recognise a place despite these factors, requiring the image comparison to be invariant or at least robust against them. Our proposed image comparison technique makes use of fast wide baseline features, namely SIFT (Lowe, 2004) and Vertical Column Segments (Goedemé et al., 2004c). These techniques compute local feature matches between the two images, invariant to the illumination. Such a local technique is also robust to occlusions. Fig. 2 shows an example of the found correspondences using these two kinds of features. These matches were found in less than half a second on up-to-date hardware and $640 \times 480$ images.

The required image comparison measure (visual distance) must be inverse proportional to the number of matches, relative to the average number of features found in the images. Hence the first two factors in equation 4. But, also the difference in relative configuration of the matches must be taken in account. Therefore, we first compute a global angular alignment of the images by computing the average angle difference of the matches. The visual distance is now also made proportional to the average angle difference of the features after this global alignment.

$$
d_{V}=\frac{1}{N} \cdot \frac{n_{1}+n_{2}}{2} \cdot \frac{\sum\left|\Delta \alpha_{i}\right|}{N}
$$

where $N$ corresponds to the number of matches found, $n_{i}$ the number of extracted features in image $i, \Delta \alpha_{i}$ the angle difference for one match after global alignment.

\subsection{Image clustering}

We define a topological map as a graph; a set of places is connected by links denoting possible transitions from one place to another. Each place is rep- 


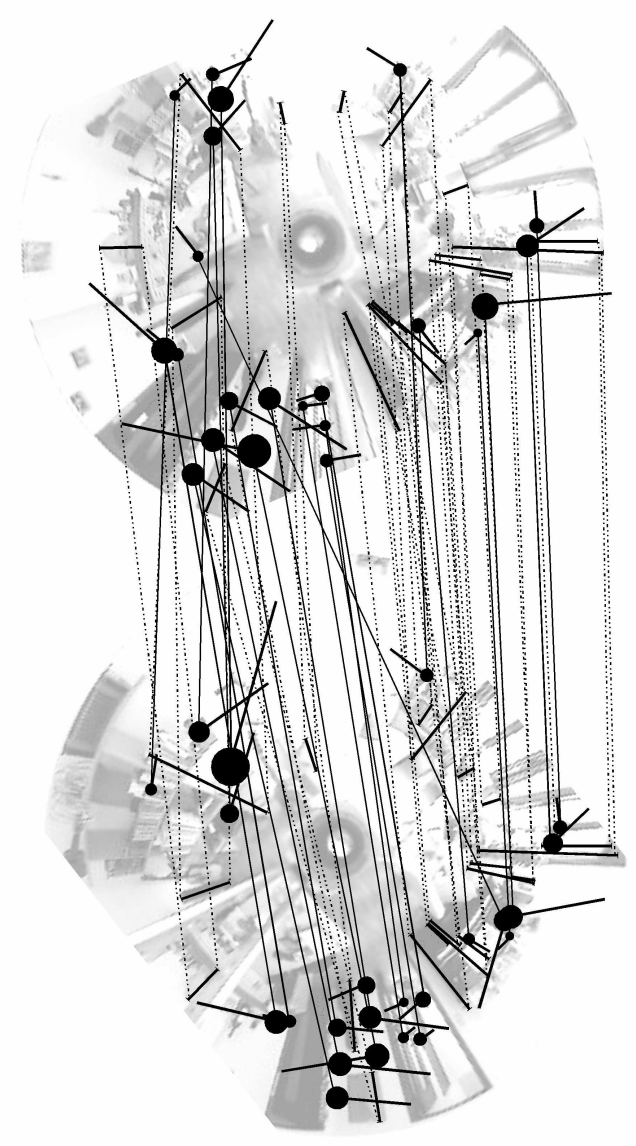

Figure 2: A pair of omnidirectional images, superimposed with corresponding column segments (radial lines, matches indicated with dotted line) and SIFT features (circles with tail, matches with continues line). The images are rotated for optimal visibility of the matches.

resented by one prototype image, and transitions between places can be done by visual servoing from one place towards the prototype of the neighbouring place. Such a visual servoing step (as we described in (Goedemé et al., 2005)) imposes a maximum visual distance between places.

The dots in the sketch figure 3 denote places where images are taken. Because they were taken at constant time intervals and the robot did not drive at a constant speed, they are not evenly spread. We perform an agglomerative clustering with complete linkage based on the visual distance (equation 4) on all the images, yielding the ellipse shaped clusters in fig. 3 . The black line shows the exploration path as driven by the robot.

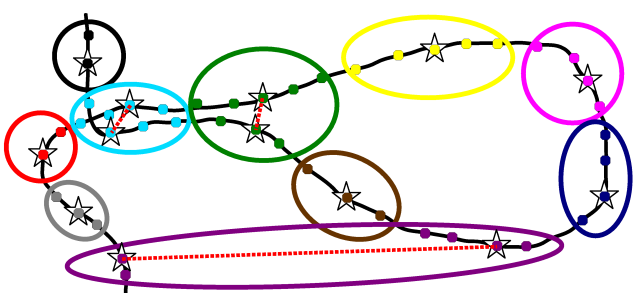

Figure 3: Example for the image clustering and hypothesis formulation algorithms. Dots are image positions, black is exploration path, clusters are visualised with ellipses, prototypes of (sub)clusters with a star. Hypotheses are denoted by a dotted red line.

\subsection{Hypothesis formulation}

As can be seen in the example (fig. 3), not all image groups nicely cover one distinct place. This is due to self-similarities, or distinct places in the environment that are different but look alike and thus yield a small visual distance between them.

For each of the clusters, we can define one or more subclusters. Images within one cluster who are linked by exploration path connections are grouped together. For each of these subclusters a prototype image is chosen as the medoid ${ }^{2}$ based on the visual distance, denoted as a star in the figure.

As can be seen in the example, clusters containing more than one subcluster can be one of two possibilities:

- real loop closings, i.e. the robot is revisiting a place and detected that it looks alike.

- erroneous self-similarities, i.e. distinct places that look alike to the system.

For each pair of these subclusters within the same cluster, we define a loop closing hypothesis $H$, which states that if $H=$ true, the two subclusters describe the same physical place and must be merged together. We will use Dempster-Shafer theory to collect evidence about each of these hypotheses.

\subsection{Dempster-Shafer evidence collection}

For each of the hypotheses defined in the previous step, a decision must be made if it was correct or wrong. Figure 4 illustrates four possibilities for one hypothesis. We observe that a hypothesis has more chance to be true if there are more hypotheses in the neighbourhood, like in case $a$ and $b$. If no neighbouring hypotheses are present $(c, d)$, no more evidence

\footnotetext{
${ }^{2}$ The medoid of a cluster is analogous to the centroid, but uses the median operator instead of the average.
} 
can be found and no decision can be made based on this data.
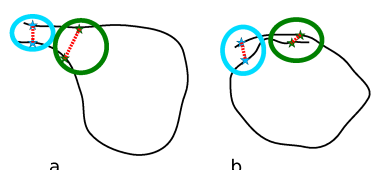
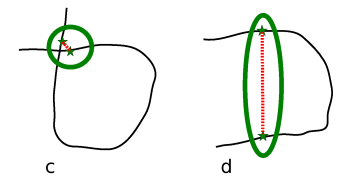

Figure 4: Four topological possibilities for one hypothesis.

We conclude that for a certain hypothesis, a neighbouring hypothesis adds evidence to it. It is clear that, the further away this neighbour is from the hypothesis, the less certain the given evidence is. We chose to model this subjective uncertainty by means of the ignorance notion in Dempster-Shafer theory. That is why we define an ignorance function containing the distance between two hypotheses $H_{a}$ and $H_{b}$ :

$\xi\left(H_{a}, H_{b}\right)= \begin{cases}1-\sin \left(\frac{d_{H}\left(H_{a}, H_{b}\right) \pi}{2 d_{t h}}\right) & \left(d_{H} \leq d_{t h}\right) \\ 0 & \left(d_{H}>d_{t h}\right)\end{cases}$

where $d_{t h}$ is a distance threshold and $d_{H}\left(H_{a}, H_{b}\right)$ is the sum of the distances between the two pairs of prototypes of both hypotheses, measured in number of exploration images.

To gather aleatory evidence, we look at the visual similarity of both subcluster prototypes, normalised by the standard deviation of the intra-subcluster visual similarities:

$$
s_{V}\left(H_{a}\right)=\frac{s_{V}\left(\operatorname{prot}_{a 1}, \operatorname{prot}_{a 2}\right)}{\sigma_{\text {subclus }}\left(s_{V}\right)},
$$

Where the visual similarity $s_{V}$ is derived from the visual distance, defined in equation 4.

Each neighbouring hypothesis $H_{b}$ yields the following set of Dempster-Shafer masses, to be combined with the masses of the hypothesis $H_{a}$ itself:

$$
\begin{array}{ll}
m(\{\emptyset\}) & =0 \\
m\left(\left\{H_{a}\right\}\right) & =s_{V}\left(H_{b}\right) \xi\left(H_{a}, H_{b}\right) \\
m\left(\left\{\neg H_{a}\right\}\right) & =\left(1-s_{V}\left(H_{b}\right)\right) \xi\left(H_{a}, H_{b}\right) \\
m\left(\left\{H_{a}, \neg H_{a}\right\}\right) & =1-\xi\left(H_{a}, H_{b}\right)
\end{array}
$$

Hypothesis masses are initialised with the visual similarity of its subcluster prototypes and a initial ignorance value ( 0.25 in our experiments), which models its influenceability by neighbours.

\subsection{Hypothesis decision}

After combination of each hypothesis's mass set with the evidence given by neighbouring hypotheses (up to a maximum distance $d_{t h}$ ), a decision must be made if this hypothesis was correct and thus if the subclusters must be united into one place or not.

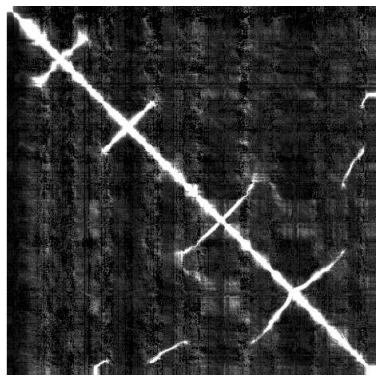

Figure 5: Matrix showing the visual similarities between the images of the experiment.

Unfortunately, as stated above, only positive evidence can be collected, because we can not gather more information about totally isolated hypotheses (like $c$ and $d$ in fig. 4). This not too bad, because of different reasons. Firstly, the chance for correct, but isolated hypothesis (case $c$ ) is low in typical cases. Also, adding erroneous loop closings ( $c$ and $d$ ) will yield an incorrect topological map, whereas leaving them out will keep the map useful for navigation, but a bit less complete. Of course, new data about these places can be acquired later, during navigation.

Important is to remind that the computed Dempster-Shafer masses can not directly be interpreted as probabilities. That is why we use equation 3 to compute the support and plausibility of each hypothesis after evidence collection. Because these values define a confidence interval for the real probability, a hypothesis can be accepted if the lower bound (the support) is greater than a threshold.

After this decision, a final topological map can be built. Subclusters connected with accepted hypotheses are merged into one place, and a new medoid is computed as prototype of it. For hypotheses that are not accepted, two distinct places should be constructed.

\section{EXPERIMENTS}

With the camera system mounted on a electric wheel chair (see fig. 1), we drove around in a complex natural environment, being our office floor. $463 \mathrm{im}$ ages were recorded during this path of $275 \mathrm{~m}$ length. Figure 6 shows a map of the environment. It can be seen that the path visits several offices and corridors more than once, generating the possibility for a lot of loop closing hypotheses. Figure 7 gives a few typical images acquired.

Between each pair of images, fast wide baseline features are matched and the proposed visual distance measure is computed, yielding the similarity matrix visualised in fig. 5. Based on this, the images are 


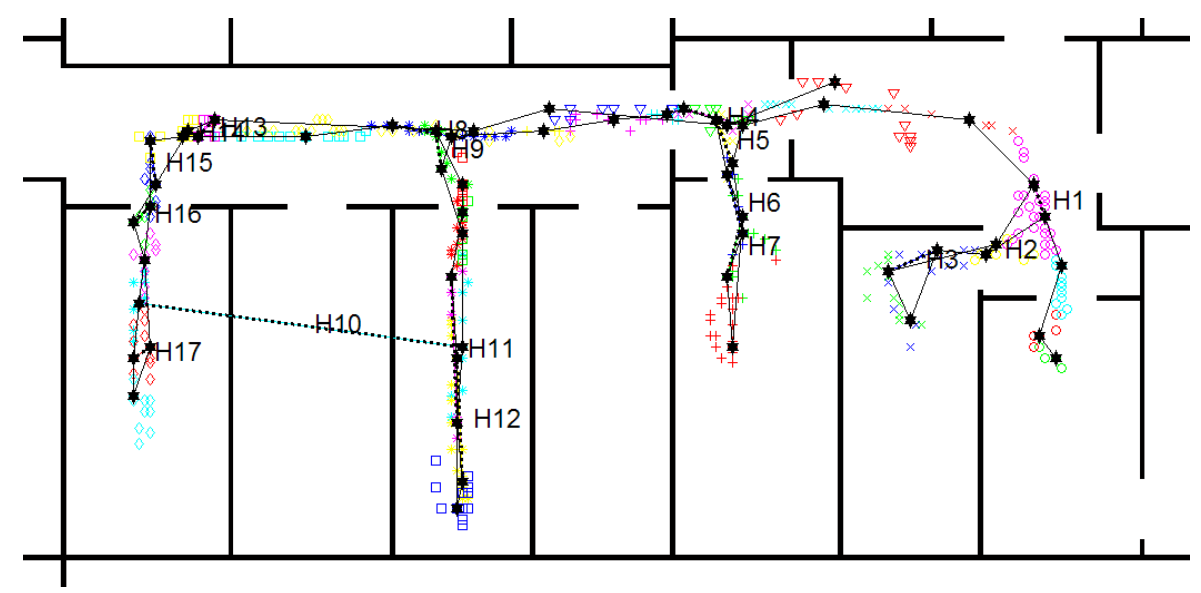

Figure 6: Right: Map of the experiment. Scattered datapoints are image positions, shown in various symbols denoting clustering. Stars are (sub)clusters. The exploration path is visualised with thin black lines, hypotheses with thick dotted lines.

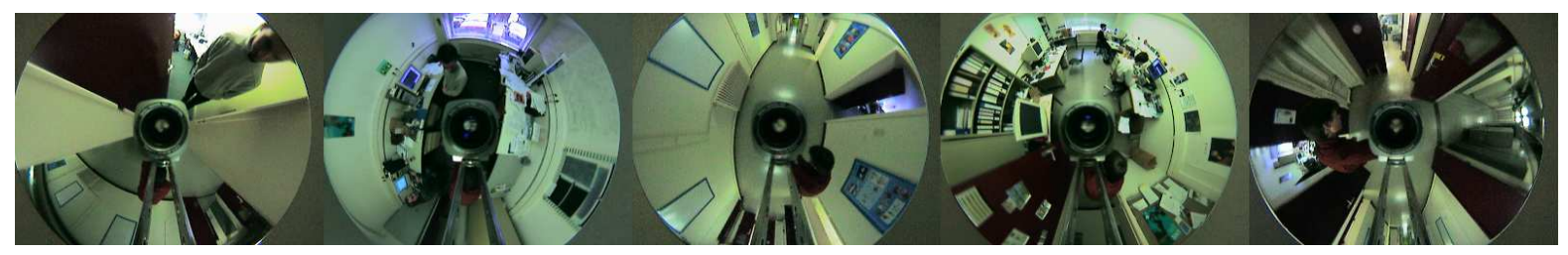

Figure 7: Images 256, 315, 345, 388 and 430 of the experiment.

clustered as shown with different symbols in fig. 6, resulting in 38 clusters. For each subcluster, a prototype is chosen denoted by a black star. Between subclusters within one cluster, hypotheses are formulated, denoted by thick black dotted lines.

As can be seen in the map, all but one hypothesis (number 10) are correct. This is clearly a selfsimilarity in the environment, the two offices do not differ enough in appearance. Figure 8 gives the Dempster-Shafer masses of each hypothesis before and after evidence collection. It is clear that after evidence combination, we have more reason to reject hypothesis 10 .

The other subclusters can be merged, resulting in the final topological map, shown in figure 9.

\section{CONCLUSION}

This paper described a method to find topological loop closings in a sequence of images taken in a natural environment. The result is a vision-based topological map which can be used for localisation, path planning and navigation.

Firstly, a robust visual distance was presented. Making use of state-of-the-art wide baseline matching techniques, this enables the recognition of places
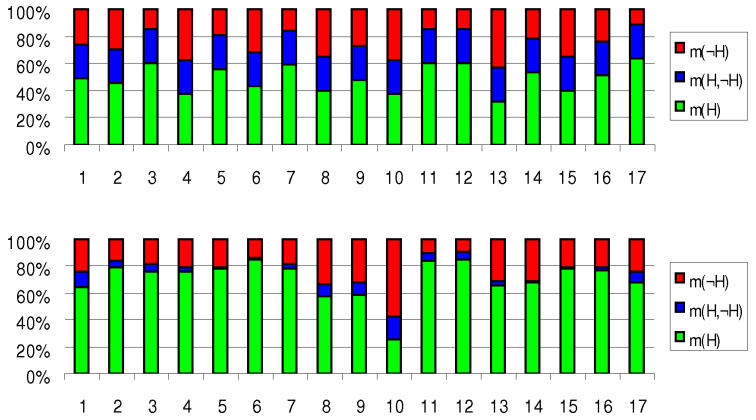

Figure 8: Dempster-Shafer masses for each of the hypotheses, before (above) and after (below) evidence collection.

despite changes in viewpoint, illumination, and the presence of occlusions.

Secondly, a mathematical model is presented to solve the problem of self-similarities, i.e. places in the environment that look alike but are different. This approach uses Dempster-Shafer probability theory to combine evidence of neighbouring loop closing hypotheses.

The real-world experiments presented illustrate the performance and robustness of the approach.

Future work planned includes the introduction of even more performant visual features such as 


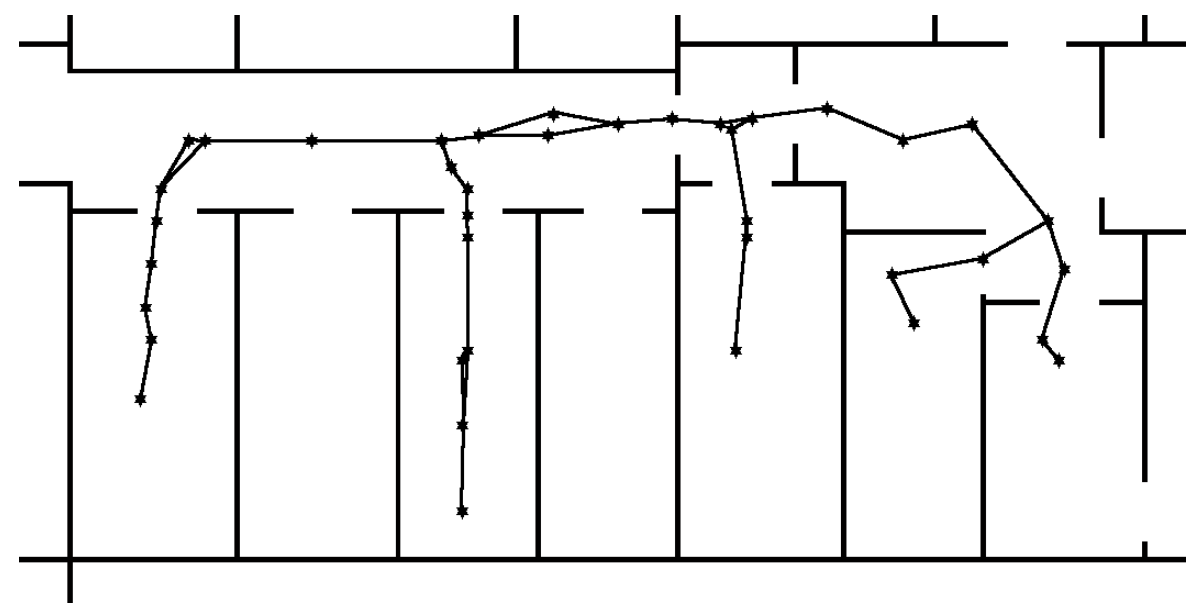

Figure 9: Final topological map. Stars denote prototypes.

SURF (Bay et al., 2006), and the on-line adaptation of the map while using it for navigation.

\section{ACKNOWLEDGEMENTS}

This work is partially supported by the InterUniversity Attraction Poles, Office of the Prime Minister (IUAP-AMS), the Institute for the Promotion of Innovation through Science and Technology in Flanders (IWT-Vlaanderen), and the Fund for Scientific Research Flanders (FWO-Vlaanderen, Belgium).

\section{REFERENCES}

Bay, H., Tuytelaars, T., and Van Gool, L. (2006). Surf: Speeded up robust features. In European Conference on Computer Vision. In press.

Beevers, K. and Huang, W. (2005). Loop closing in topological maps. In ICRA.

Chen, C. and Wang, H. (2005). Appearance-based topological bayesian inference for loop-closing detection in cross-country environment. In IROS, pages 322-327.

Dempster, A. P. (1967). Upper and lower probabilities induced by a multivalued mapping. In The Annals of Statistics (28), pages 325-339.

Goedemé, T., Nuttin, M., Tuytelaars, T., and Van Gool, L. (2004a). Markerless computer vision based localization using automatically generated topological maps. In European Navigation Conference GNSS.

Goedemé, T., Nuttin, M., Tuytelaars, T., and Van Gool, L. (2004b). Vision based intelligent wheelchair control: the role of vision and inertial sensing in topological navigation. In Journal of Robotic Systems, 21(2), pages 85-94.
Goedemé, T., Tuytelaars, T., and Van Gool, L. (2004c). Fast wide baseline matching with constrained camera position. In Conference on Computer Vision and Pattern Recognition, pages 24-29.

Goedemé, T., Tuytelaars, T., Vanacker, G., Nuttin, M., and Van Gool, L. (2005). Feature based omnidirectional sparse visual path following. In International Conference on Intelligent Robots and Systems, IROS 2005, pages 1003-1008.

Koenig, S. and Simmons, R. (1996). Unsupervised learning of probabilistic models for robot navigation. In ICRA.

Lowe, D. (2004). Distinctive image features from scaleinvariant keypoints. In IJCV (60), pages 91-110.

Nagatani, K., Choset, H., and Thrun, S. (1998). Towards exact localization without explicit localization with the generalized voronoi graph. In ICRA, pages 342-348.

Ranganathan, A., Menegatti, E., and Dellaert, F. (2005). Bayesian inference in the space of topological maps. In IEEE Transactions on Robotics.

Shafer, G. (1976). A Mathematical Theory of Evidence. Princeton University Press.

Shatkay, H. and Kaelbling, L. P. (1997). Learning topological maps with weak local odometric information. In IJCAI (2), pages 920-929.

Tapus, A. and Siegwart, R. (2005). Incremental robot mapping with fingerprints of places. In IROS.

Tolman, E. C. (1948). Cognitive maps in rats and men. In Psychological Review (55), pages 189-208.

Tomatis, N., Nourbakhsh, I. R., and Siegwart, R. (2002). Hybrid simultaneous localization and map building: Closing the loop with multi-hypotheses tracking. In ICRA, pages 2749-2754.

Wahlgren, C. and Duckett, T. (2005). Topological mapping for mobile robots using omnidirectional vision. In SWAR.

Zivkovic, Z., Bakker, B., and Kröse, B. (2005). Hierarchical map building using visual landmarks and geometric constraints. In IROS, pages 7-12. 